\title{
Bifocal Tuberculosis with Adalimumab Revealed by Laryngeal Involvement: Case Report
}

\author{
Najlae El Ouardi ${ }^{1 *}$ (D), Laila Taoubane ${ }^{1}$, Adil Zegmout ${ }^{2}$, Marouane Balouki ${ }^{3}$, Siham Sadni ${ }^{1}$, \\ Lahsen Achemlal ${ }^{1}$
}

\begin{abstract}
Introduction. Anti-TNF treatment has transformed the treatment of chronic inflammatory rheumatism. Although the therapy can be highly effective, anti-TNF drugs are associated with an increased risk of tuberculosis, especially extra-pulmonary tuberculosis.

Laryngeal tuberculosis is rare and its symptoms are not specific. Laryngeal tuberculosis is often secondary to another localization, particularly pulmonary. In the use of anti-TNF therapy, its development is unusual.

Case report. We report a case of bifocal tuberculosis: laryngeal and pulmonary tuberculosis revealed by laryngeal involvement in a patient aged 41 years with axial spondylarthritis treated with Adalimumab.

Conclusion. This presentation highlights the importance to consider the rare possibility of laryngeal tuberculosis in the presence of atypical otorhinolaryngologic signs under anti-TNF therapy and underlines the importance of looking for other tuberculosis involvement.
\end{abstract}

\section{Keywords}

Spondyloarthritis; Bifocal Tuberculosis; Laryngeal Tuberculosis; Adalimumab

${ }^{1}$ Rheumatology Department, Mohammed V Military Academic Hospital, Faculty of Medicine and Pharmacy, Mohammed V-Souissi University, Rabat, Morocco

${ }^{2}$ Pneumology Department, Mohammed V Military Academic Hospital, Faculty of Medicine and Pharmacy, Mohammed V-Souissi University, Rabat, Morocco

${ }^{3}$ ORL Department, Mohammed V Military Academic Hospital, Faculty of Medicine and Pharmacy, Mohammed V-Souissi University, Rabat, Morocco

*Corresponding author: doc.najlae89@gmail.com

\section{Introduction}

Anti-TNF drugs have improved the management of chronic inflammatory rheumatic diseases. However, they are not devoid of side effects, particularly infectious ones. Indeed, antiTNF drugs can reactivate latent tuberculosis, especially extrapulmonary tuberculosis. This risk is particularly increased with Infliximab and Adalimumab [1].

Laryngeal tuberculosis is rare and rarely primitive. It is often secondary to primary pulmonary localization. Its clinical presentation is often variable and not specific [2]. It should be suspected systematically when there are atypical otorhinolaryngologic symptoms under anti-TNF treatment.

The interest of this observation is to point out to the exceptional localization of tuberculosis in patients under anti-TNF drugs.

We report the original observation of bifocal laryngeal and pulmonary tuberculosis revealed by sore throat in a patient followed for spondylarthritis (SpA) under the use of Adalimumab.

\section{Observation}

Mr. H.A., 41 years old, non-smoker and non-alcoholic male patient, followed for 13 years for a severe axial SpA complicated by bilateral coxitis that required a double hip replacement surgery (double total hip replacement). Biotherapy with Etanercept (50 mg/week) was indicated due to the severity of the osteoarticular damage and the resistance to different families of non-steroidal anti-inflammatory drugs. All screening tuberculosis tests were normal, therefore Etanercept was initiated in 2014 without anti-tuberculous chemoprophylaxis. A switch to Adalimumab (40mg/15 days) was decided in 2019 since an anterior uveitis of the left eye had developed in 
the patient.

After 3 months of the introduction of Adalimumab, the patient was presented with sore throat, episodes of dysphonia and progressive difficulty in deglutition despite 2 weeks course of antibiotic and high dose of corticosteroids. The patient complained of weight loss of at least $10 \%$ in just 3 months. There were no chest or osteoarticular signs. He did not report any known contact with a case of active tuberculosis.

On examination, the patient was afebrile, looked thin with body mass index: $15.64 \mathrm{Kg} / \mathrm{m}^{2}$. There were no evident cervical Lymphadenopathies. Physical examination of the chest was normal.

Laboratory tests revealed inflammation with C-reactive protein (CRP) at $36.1 \mathrm{mg} / \mathrm{L}$, erythrocyte sedimentation rate at $35 \mathrm{~mm}$ with persistent lymphopenia $1100 / \mu 1$. Serum and urine calcium were normal. Viral serology (Epstein Bar virus, cytomegalovirus and human immunodeficiency virus) was negative. A chest X-ray was performed, revealing a normal aspect.

Both sputum microscopy for acid fast bacillus, and Quantiferon test were negative. Serum angiotensin converting enzyme was normal.

Fiberoptic nasopharyngolaryngoscopy revealed a supraglottic edema (Fig. 1) confirmed by cervical computed tomography scan (Fig. 2).

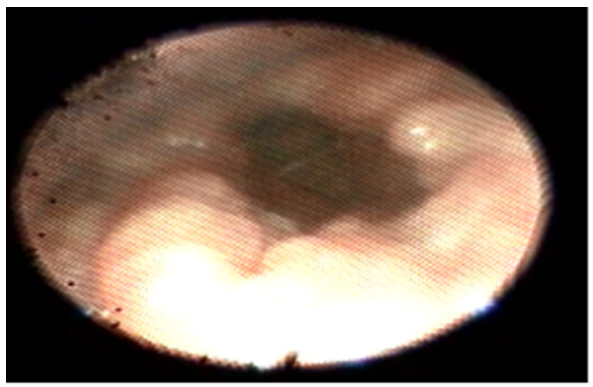

Figure 1. Laryngeal endoscopy showing a supraglottic oedema and nodular mucosa without salivary stasis or lesions suspicious of malignancy (screenshot of live laryngoscopy).

As malignancy was suspected, a laryngeal biopsy was performed. Histology revealed no malignancy but gigantocellular and epithelioid granulomas without caseous necrosis. Eso-gastrointestinal endoscopy was normal.

We've completed with thoracic computed tomography (Fig. 3), which showed a focal pulmonary condensation and micronodular infiltration. A bronchoscopy demonstrated a laryngeal translucent granules and inflammatory lesions of the right bronchus. GeneXpert test on the Bronchoalveolar lavage fluid was negative. Histological examination of the bronchial biopsy showed non-specific fibro-inflammatory lesions with no tumor lesion.

The sputum microbiological culture revealed mycobacterium tuberculosis 4 weeks later. Hence, final diagnosis was

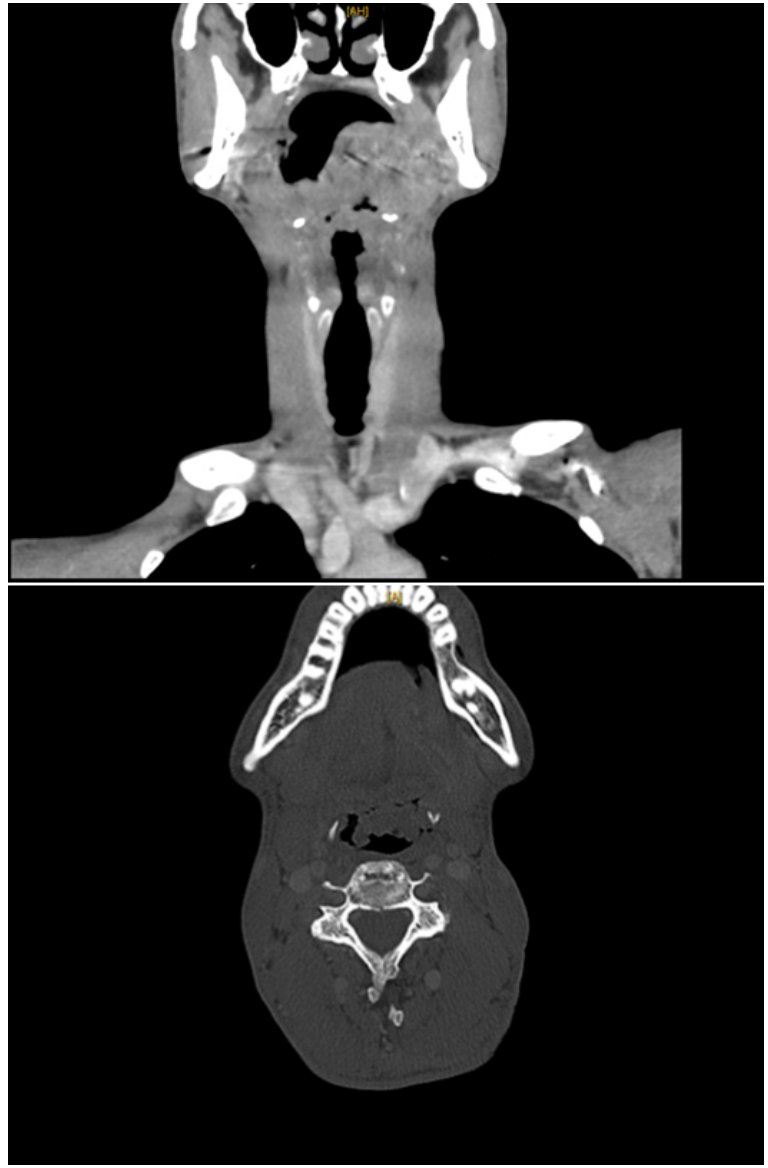

Figure 2. Cervical computed tomography showing diffuse supraglottic laryngeal edema.

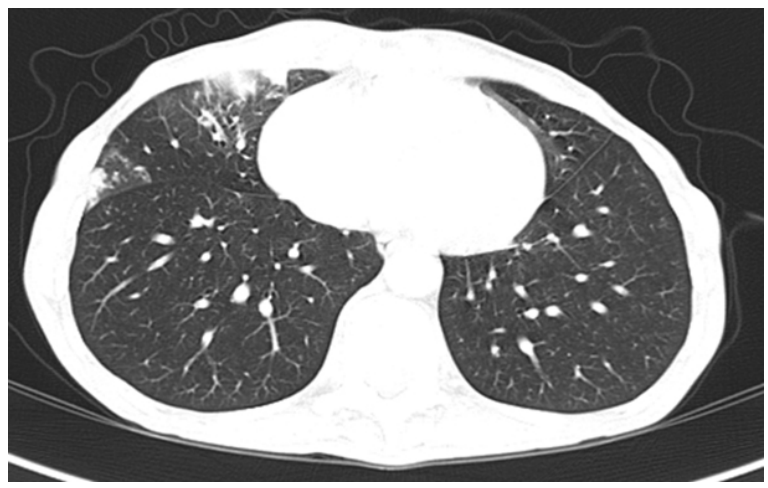

Figure 3. Thoracic computed tomography showing branching condensation surrounded by a frosted glass area and a micronodular infiltrate of the right middle pulmonary lobe.

bifocal laryngeal and pulmonary tuberculosis under Adalimumab. Adalimumab was stopped and Chemotherapy was initiated, including: 2-months of quadruple therapy: rifampicin, isoniazid, pyrazinamide and ethambutol, followed by 7 months of rifampicin and isoniazid bi-therapy.

On follow up, there were complete resolution of clini- 
cal symptoms (complete clearing of dysphagia, dysphonia and sore throat), and normalization of the biological tests (inflammation and lymphopenia). All lesions were cleared in the otorhinolaryngologic control (Fig. 4), bacilloscopic control of sputum was negative and pulmonary lesions subsided (Fig. 5).

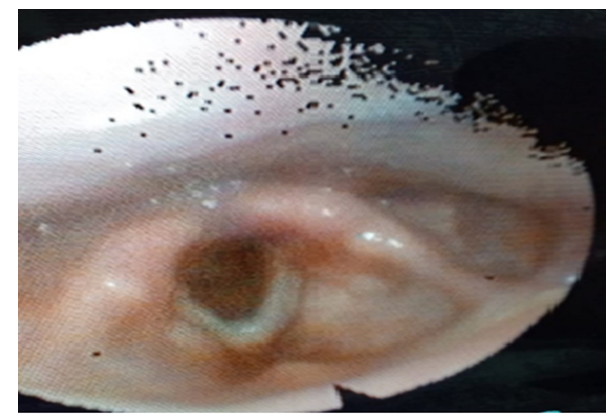

Figure 4. Laryngeal endoscopic control after 4 months of the anti-tubercular treatment. Supraglottic oedema and nodular mucosa had disappeared (screenshot of live laryngoscopy).

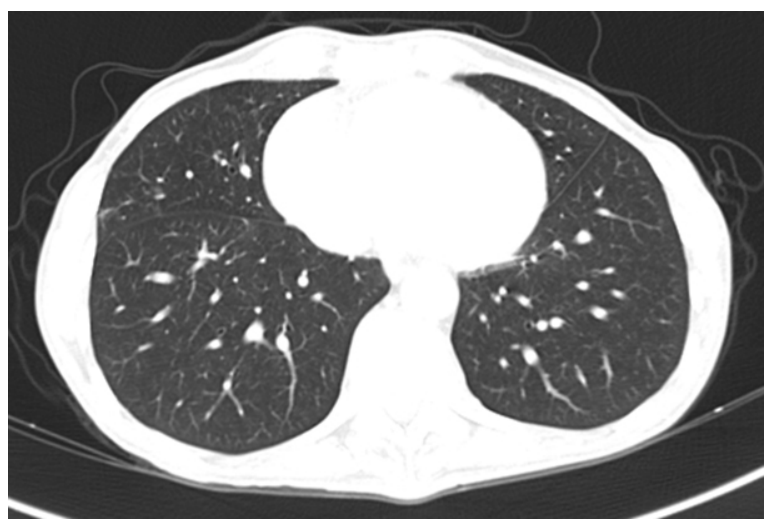

Figure 5. Control thoracic computed tomography after 4 months of the anti-tubercular treatment. Condensation and

frosted glass area of the middle pulmonary lobe had disappeared.

\section{Discussion}

Anti-TNF drugs constitute a breakthrough in the treatment of SpA. Although their efficacy, they are associated with the serious risk of developing infectious tuberculosis [1].

Anti-TNF therapy interferes negatively with the formation and the maintenance of tuberculosis granuloma, facilitating the reactivation of tuberculosis [3]. More than 50\% of tuberculosis cases developed under anti-TNF therapy, are extra-pulmonary ones [4].

The introduction of latent tuberculosis screening procedures is necessary before starting anti-TNF therapy. However, tuberculosis developed under anti-TNF drugs can still occur despite a negative initial screen $[5,6]$.
Indeed, tuberculosis in patients receiving anti TNF-therapy usually arises from the reactivation of latent infection and occurs often within the first three to eight months. Nevertheless, many cases of new tuberculosis infection under anti-TNF drugs have been reported [7].

Quantiferon test is more specific than tuberculin tests in screening for pulmonary tuberculosis. However, false negatives and false positives have been described especially in immunosuppressed patients [3].

GeneXpert is recommended by the World Health Organization in the pulmonary and extra-pulmonary tuberculosis diagnosis in both immunocompetent and immunocompromised patients $[8,9]$. It is a rapid molecular test with high performance (sensitivity and specificity) [10]. Multiple geneXpert testing on bronchoalveolar lavage fluid can improve the test sensitivity [9].

Laryngeal tuberculosis is observed very rarely $(0.5-2 \%$ of patients with tuberculosis), usually with immunocompromised diseases. It is the most common granulomatous disease of the larynx and represents less than $1 \%$ of all forms of tuberculosis $[11,12]$.

Laryngeal tuberculosis is often secondary to bronchogenic, hematogenous or lymphatic dissemination [13]. Laryngeal involvement has been observed in 15 to $37 \%$ of pulmonary tuberculosis cases, but primary involvement has been observed in only in $19 \%$ of tuberculosis cases [14]. There are only a few isolated cases in the literature [15].

Several sites may be affected: vocal cords (50-70\%), false vocal cords (40-50\%), epiglottis, subglottic region, and posterior commissure (10-15\%). Symptoms of laryngeal tuberculosis are dysphonia, hoarseness, wasting, dysphagia and odynophagia [11-16].

The lesions seen on laryngoscopy may be granulomatous, edematous, ulcerative, polyploid or non-specific $[15,16]$.

In immunocompromised patients, laryngeal tuberculosis could be confirmed in patients with chronic granulomatous inflammatory exudates and a complete recovery under antitubercular therapy even without the presence of caseation [16-19].

However, the absence of caseous necrosis raises diagnostic difficulties and can be easily confused clinically with laryngeal cancer and other granulomatosis. A biopsy is mandatory for a definitive diagnosis. Under antitubercular treatment, the clinical signs of laryngeal tuberculosis disappear within a few weeks [16].

A long-term follow-up is necessary since the laryngeal complications can occur [12].

Bifocal pulmonary and laryngeal tuberculosis under antiTNF therapy is exceptional. The literature review reports only one case developed under Adalimumab in ulcerated colitis [20].

\section{Conclusions}

Laryngeal tuberculosis under anti-TNF therapy is rare, its clinical signs are non-specific. It is often associated with 
pulmonary involvement. Thus, the suspicion of laryngeal tuberculosis requires the search for a pulmonary involvement. Our case is an unusual form of bifocal laryngeal and pulmonary tuberculosis under anti-TNF treatment revealed by laryngeal involvement.

\section{Ethical Statement}

The research was conducted according to the Declaration of Helsinki.

\section{Informed Consent}

Written informed consent was obtained from the patient.

\section{Conflict of Interest}

The authors declare that no conflicts exist.

\section{Financial Disclosure}

The authors declared no financial support.

\section{References}

[1] Tubach F, Salmon D, Ravaud P, Allanore Y, Goupille $\mathrm{P}$, Bréban M, et al. Risk of tuberculosis is higher with anti-tumor necrosis factor monoclonal antibody therapy than with soluble tumor necrosis factor receptor therapy: The three-year prospective french research axed on tolerance of biotherapies registry. Arthritis \& Rheumatism [Internet]. 2009 Jul;60(7):1884-1894. Available from: https://doi.org/10.1002/art.24632

[2] Agarwal R, Gupta L, Singh M, Yashaswini N, Saxena A, Khurana N, et al. Primary Laryngeal Tuberculosis: A Series of 15 Cases. Head and Neck Pathology [Internet]. 2018 Sep 24;13(3):339-343. Available from: https://doi.org/10.1007/s12105-018-0970-y

[3] Abreu C, Magro F, Santos-Antunes J, Pilão A, RodriguesPinto E, Bernardes J, et al. Tuberculosis in anti-TNF- $\alpha$ treated patients remains a problem in countries with an intermediate incidence: Analysis of 25 patients matched with a control population. Journal of Crohn's and Colitis [Internet]. 2013 Nov;7(10):e486-e492. Available from: https://doi.org/10.1016/j.crohns.2013.03.004

[4] BTS recommendations for assessing risk and for managing Mycobacterium tuberculosis infection and disease in patients due to start anti-TNF- treatment. Thorax [Internet]. 2005 Oct 1;60(10):800-805. Available from: https://doi.org/10.1136/thx.2005.046797

[5] Jauregui-Amezaga A, Turon F, Ordás I, Gallego M, Feu F, Ricart E, et al. Risk of developing tuberculosis under anti-TNF treatment despite latent infection screening. Journal of Crohn's and Colitis
[Internet]. 2013 Apr;7(3):208-212. Available from: https://doi.org/10.1016/j.crohns.2012.05.012

[6] Kedia S, Mouli VP, Kamat N, Sankar J, Ananthakrishnan A, Makharia G, et al. Risk of Tuberculosis in Patients With Inflammatory Bowel Disease on Infliximab or Adalimumab Is Dependent on the Local Disease Burden of Tuberculosis. The American Journal of Gastroenterology [Internet]. 2020 Mar;115(3):340-349. Available from: https://doi.org/10.14309/ajg.0000000000000527

[7] Abitbol Y, Laharie D, Cosnes J, Allez M, Nancey S, Amiot A, et al. Negative Screening Does Not Rule Out the Risk of Tuberculosis in Patients with Inflammatory Bowel Disease Undergoing Anti-TNF Treatment: A Descriptive Study on the GETAID Cohort. Journal of Crohn's and Colitis [Internet]. 2016 Jul 11;10(10):1179-1185. Available from: https://doi.org/10.1093/ecco-jcc/jjw129

[8] Mechal Y, Benaissa E, El mrimar N, Benlahlou Y, Bssaibis F, Zegmout A, et al. Evaluation of GeneXpert MTB/RIF system performances in the diagnosis of extrapulmonary tuberculosis. BMC Infectious Diseases [Internet]. 2019 Dec;19(1). Available from: https://doi.org/10.1186/s12879-019-4687-7

[9] Yeong C, Byrne AL, Cho J-G, Sintchenko V, Crighton T, Marais BJ. Use of GeneXpert MTB/RIF on a single pooled sputum specimen to exclude pulmonary tuberculosis among hospital inpatients placed in respiratory isolation. International Journal of Infectious Diseases [Internet]. 2020 Mar;92:175-180. Available from: https://doi.org/10.1016/j.ijid.2019.12.024

[10] Sharma SK, Mohan A, Sharma A. Miliary tuberculosis: A new look at an old foe. Journal of Clinical Tuberculosis and Other Mycobacterial Diseases [Internet]. 2016 May;3:13-27. Available from: https://doi.org/10.1016/j.jctube.2016.03.003

[11] Benwill JL, Sarria JC. Laryngeal tuberculosis in the United States of America: A forgotten disease. Scandinavian Journal of Infectious Diseases [Internet]. 2014 Feb 7;46(4):241-249. Available from: https://doi.org/10.3109/00365548.2013.877157

[12] Avula A, Ngu S, Mansour W, Gurala D, Maroun R. A Case of Laryngeal Tuberculosis, Endobronchial Tuberculosis and Pulmonary Tuberculosis Coexistent in an Immunocompetent Host. Cureus [Internet]. 2020 Sep 29. Available from: https://doi.org/10.7759/cureus.10713

[13] Lim J-Y, Kim K-M, Choi EC, Kim Y-H, Kim HS, Choi HS. Current clinical propensity of laryngeal tuberculosis: review of 60 cases. European Archives of Oto-RhinoLaryngology [Internet]. 2006 Jul 12;263(9):838-842. Available from: https://doi.org/10.1007/s00405-0060063-5 
[14] Hone, Timon, Harney, Donnelly. Laryngeal tuberculosis: an important diagnosis. The Journal of Laryngology \& Otology [Internet]. $2000 \mathrm{Nov} ; 114(11): 878-880$. Available from: https://doi.org/10.1258/0022215001904220

[15] Al-Tawfiq JA. Multifocal systemic tuberculosis: the many faces of an old nemesis. Med Sci Monit. 2007 Apr;13(4) CS56-60. Available from: https://www.medscimonit.com/

[16] Swain SK, Behera IC, Sahu MC. Primary Laryngeal Tuberculosis: Our Experiences at a Tertiary Care Teaching Hospital in Eastern India. Journal of Voice [Internet]. 2019 Sep;33(5):812.e9-812.e14. Available from: https://doi.org/10.1016/j.jvoice.2018.04.010

[17] Hurtado Alegre JL, Trigoso Gutierrez A, Matos Prado E, Huaringa Marcelo J. Laryngeal Tuberculosis in Pregnant Women: A Case Report and Review of the Literature. Cureus [Internet]. 2018 Nov 5. Available from: https://doi.org/10.7759/cureus.3545

[18] Topak M, Oysu C, Yelken K, Sahin-Yilmaz A, Kulekci M. Laryngeal involvement in patients with active pul- monary tuberculosis. European Archives of Oto-RhinoLaryngology [Internet]. 2007 Oct 6;265(3):327-330. Available from: https://doi.org/10.1007/s00405-0070459-x

[19] Lodha J, Sharma A, Virmani N, Bihani A, Dabholkar J. Secondary laryngeal tuberculosis revisited. Lung India [Internet]. 2015;32(5):462-464. Available from: https://doi.org/10.4103/0970-2113.164163

[20] Bosch A, Conrad A, Fuchsmann C, Prot B, Dumitrescu $\mathrm{O}$, Ferry T, et al. Severe laryngeal and pulmonary tuberculosis under anti-TNF- $\alpha$ therapy. International Journal of Infectious Diseases [Internet]. 2018 Jun;71:65-66. Available from: https://doi.org/10.1016/j.ijid.2018.04.797

Received: $2020-10-18$

Revised: $2020-11-27$

Accepted: 2020-12-05 